\title{
CONTRATO Y DIBUJO INÉDITO DE UNAS LÁMPARAS DE PLATA PARA LA CATEDRAL DE CORDOBA ${ }^{1}$
}

\author{
MARÍA DEL AMOR RODRÍGUEZ MIRANDA \\ Universidad de Córdoba
}

\begin{abstract}
Resumen
En este trabajo se dan a conocer unos documentos relacionados con el encargo y ejecución de dos lámparas de plata, destinadas a la Capilla de Villaviciosa y la Capilla del Sagrario, ambas en la Catedral cordobesa. Este compromiso fue instituido con cargo a una Obra Pía fundada por don Juan de Valderrama, en la había quedado establecida la elaboración de estas piezas en el año 1647 por el platero cordobés Gonzalo de Alcántara. Se presenta además el dibujo original realizado por el maestro del diseño de las mismas.
\end{abstract}

Palabras clave

Contrato, inédito, diseño, Catedral de Córdoba, lámpara, siglo XVII, Obra Pía.

\section{CONTRACT AND UNPUBLISHED DRAWING OF SOME SILVER LAMPS FOR THE CATHEDRAL OF CORDOBA}

Abstract

In this work there are announced several documents around the order and execution of two silver lamps, destined to Villaviciosa's chapel and the Chapel of the Sacrarium, both in the Cathedral cordobesa. This order was realized by post to the Pious Work, founded dy don Juan de Valderrama, in her there had remained established the making of these pieces in the year 1647 with the silversmith native of Cordoba Gonzalo de Alcántara. The original drawing made by the master of the same in also presented.

Keywords

Contract, unpublished, drawing, Cathedral, Córdoba, lamp, XVIIth, Pious Work.

\footnotetext{
${ }^{1}$ Este trabajo se inscribe en el marco del Grupo de Investigación y Tecnología de Bienes Culturales (INTECBIC), HUM-428.
} 


\section{Consideraciones generales sobre las lámparas del siglo XVII}

La lámpara como objeto litúrgico fue una pieza muy demandada y utilizada en la Edad Moderna. Su uso se remonta en el tiempo hasta el Antiguo Testamento. El libro del Éxodo dispone que una lámpara debe arder continuamente junto al tabernáculo ${ }^{2}$. Desde entonces ha estado presente en reglamentos y escritos relacionados con la disposición del culto y los rituales eclesiásticos, como las ordenanzas del abad Bernardo de Cluny $^{3}$, las Constituciones del Císter ${ }^{4}$ o las Caeremoniale Episcoporum, cuya primera redacción se hizo durante el papado de Sixto V en 1587. Estos libros están compuestos por tres grandes apartados que contienen los ritos y ceremonias católicas. En los capítulos XII al XIV del libro I quedan definidos los ornamentos de la iglesia y del trono del obispo. Poco después, en 1600, Clemente VIII los corrigió y los compiló. Por lo que fue relativamente habitual que iglesias, catedrales y otros templos menores se proveyeran de este tipo de obras. Encontrar ejemplares conservados en los ajuares eclesiásticos es relativamente frecuente. En los archivos son frecuentes los contratos de ejecución a partir del siglo XVII, pero no es tan habitual hallar el dibujo que acompañe al contrato -en especial de esta centuria-, como es este caso ${ }^{5}$.

Estas piezas debían cumplir un objetivo muy explícito, que consistía en mostrar al fiel que ese era el lugar reservado a la presencia de Cristo. Normalmente eran encargadas por titulares de patronatos o capellanías, hermanos mayores de hermandades y cofradías, nobles locales o miembros de la élite eclesiástica. Con ello, no sólo deseaban iluminar ese espacio sagrado, sino que su nombre fuera reconocido al haber hecho una acción benefactora de esa envergadura.

El panorama de la producción argéntea de lámparas en la primera mitad del seiscientos fue bastante prolífico en Córdoba y su provincia. Los templos que adquirieron algún ejemplar o recibieron una de ellas a cuenta de alguna de esas donaciones mencionadas en el párrafo anterior fueron numerosos. Era también usual que existiera una persona encargada sólo y exclusivamente de su mantenimiento, del aceite que necesitara y de todos sus cuidados ${ }^{6}$. Un ejemplo de ello se encuentra en la catedral cordobesa, donde dicha figura quedaba definida en los estatutos catedralicios ${ }^{7}$. La primera vez que se encarga un ejemplar de plata en Córdoba será en el siglo XVII para la Capilla de Villaviciosa, precisamente una de las capillas para las que estaba dedicada una de las dos lámparas que se encargan en este estudio. En 1604 se pedirá una para la parroquial de Bujalance, que fue terminada en $1625^{8}$. De 1610 quedó constancia de la realización de otra para la cofradía del Rosario de Luque ${ }^{9}$, que no se ha conservado. La congregación del Rosario y de Ánimas de Priego de Córdoba contratará la suya en $1630^{10}$, la del Rosario de Córdoba dos años después y el Colegio de la Piedad de Córdoba para su capilla del Santísimo en $1637^{11}$. Pero de todas ellas, la realizada por Martín Sánchez de la Cruz para la catedral cordobesa en 1629 y que pende de su altar mayor será la más monumental ${ }^{12}$. Los encargados de la realización de ellas serán los plateros más destacados del momento, como Jerónimo de la Cruz o Pedro Sánchez de Luque, entre otros. Algunas de estas piezas documentadas se han conservado, como la de Bujalance ${ }^{13}$ o Priego

\footnotetext{
2 Éxodo, 27, 20-21.

${ }^{3}$ López, 2005: 136.

${ }^{4}$ Nieto/Moreno, 1993: 155.

${ }^{5}$ Se referencian algunos artículos en la bibliografía, destacando los que estudian lámparas de plata y los que tratan sobre dibujos y plateros del siglo XVII.

${ }^{6}$ Las funciones y el significado de las lámparas quedan definidas en: Rivas, 2003: 515-536.

${ }^{7}$ Fresnada, 1577: 96-97.

8 Valverde, 1963: 31 y 40; y Ortiz, 1986: 236.

${ }^{9}$ Torre, 1983: 103.

10 Torre, 1983: 143.

11 Torre, 1983: 148 y 173.

${ }^{12}$ Esta obra ha sido estudiada por diferentes autores, de los que hay que destacar que ya en el año 1946 se le dedicara el primer artículo monográfico: Nieto/Moreno, 1993: 184-185; Romero, 1946; Valverde, 1973a; y Ortiz, 1986: 239-240.

${ }^{13}$ Existe una lámpara en el actual sagrario que se corresponde con la estética de la primera mitad del siglo XVII, de la que tanto Ortiz Juárez como Nieto Cumplido y Moreno Cuadro afirman que lleva los punzones de Simón Pérez de Tapia, lo que no ha podido ser comprobado ya que en la actualidad dichas marcas han
} 
de Córdoba; pero otras no. Existen además otras obras no documentadas, que se han preservado en diferentes localidades cordobesas, como las de la parroquia de Santiago y el oratorio de Nuestra Señora de la Paz de Montilla, las de la capilla del Santísimo Sacramento del templo de Nuestra Señora del Soterraño de Aguilar de la Frontera o varias del Sagrario de Nuestra Señora de la Asunción de Priego de Córdoba.

En un primer estudio se pueden ver claramente que hay ciertas características estructurales que se repiten. Se componen de una boya con un solo cuerpo y elemento pinjante colgante. Entre los recursos decorativos destacan los gallones, los óvalos en resalte y las tornapuntas cinceladas. Las diferencias van a consistir en disponer de más o menos ingredientes ornamentales, por lo que se pueden encontrar lámparas con abundante ornato, otras donde se combinan los espacios adornados y sin ellos, y otras mucho más sencillas. El borde suele ser plano y liso, sobresaliendo de la boya y en él se insertan recursos figurativos o vegetales horadados de los que parten las cadenas. Tienen eslabones moldurados y rematan en un manípulo con argolla para colgarla. Este manípulo puede estar más o menos modelado. Peculiaridades que se pueden ver tanto en la monumental lámpara catedralicia como en la que se estudia en el presente texto, el cual es uno de los ejemplares cordobeses más sencillos y a la vez elegantes de esta tipología ${ }^{14}$.

\section{El contrato de las lámparas, su dibujo y sus benefactores}

El 13 de septiembre de 1639, don Juan de Valderrama ${ }^{15}$, familiar del Santo Oficio ${ }^{16}$, había firmado su testamento ante Rodrigo de Molina. En dicho otorgamiento se decía que era hijo de Diego Rodríguez Valderrama y de doña Leonor Ramírez de Velasco, ambos procedentes de Granada. Una de las disposiciones del documento era que se creara una Obra Pía y que se destinaran parte de sus bienes al socorro de viudas, huérfanas y pobres ${ }^{17}$. Pero, además, había otro tipo de preceptos. Entre ellas destaca la orden de realizar dos lámparas de plata, que debían ser iguales y que iban a ser destinadas una a la capilla de Nuestra Señora de Villaviciosa y otra a la capilla del Sagrario, ambos sitios ubicados en el interior de la catedral cordobesa. Don Juan morirá el 27 de octubre de 1639 sin haber visto construidas las lámparas: "D. Juan de Valderrama, familiar del Santo Oficio, vecino de Córdoba, hijo de Diego Rodríguez. Valderrama, familiar del Santo Oficio de Granada y doña Leonor Ramírez de Velasco, naturales de Granada, dispone que se funde una Obra Pia con parte de su patrimonio destinada a socorrer a viudas, huérfanas y pobres y se digan misas por su alma en diferentes conventos de Córdoba perpetuamente. Del mismo modo dispone que se hicieran dos lámparas de plata, una para la capilla del Sagrario y otra para la de Villaviciosa y que se costee el aceite de las mismas perpetuamente"18.

En el apartado anterior se destacó el hecho de que este elemento de iluminación era frecuente que procediera de una donación y que iban destinados a los sagrarios, pero también a ciertas capillas de renombre, como es este caso. La actual parroquia del Sagrario fue instaurada como capilla a finales del siglo XVI en otra anterior dedicada al apóstol Santiago, fundada en 1330 en el ángulo suroeste de la antigua Mezquita. Es un espacio grandioso que ocupa tres naves de la ampliación de Almanzor ${ }^{19}$ y que se la ha llegado a denominar la "capilla sixtina cordobesa". Posee este templo interno un gran ajuar litúrgico, aunque no se ha conservado ninguna lámpara de este período ${ }^{20} \mathrm{o}$ su ubicación es diferente en la actualidad y

desaparecido, por lo que es muy difícil saber sí esta lámpara es la de Simón Pérez de Tapia o la que en 1625 hizo Pedro Sánchez de Luque. Ortiz, 1986: 249; y Nieto/Moreno, 1993: 186.

${ }^{14}$ Romero, 1946: 85-96.

15 Valverde, 2000: 209.

${ }^{16}$ El profesor Valverde identificó un Juan Antonio Valderrama, familiar del Santo Oficio en el año 1659, que era hijo de Antonio y de María del Rosal, hija ésta a su vez de otro familiar del Santo Oficio, Juan del Rosal. Tenía un hijo Juan Antonio que se llamaba Antonio Valderrama, que también era familiar y cuyo expediente era el $n^{\circ} 5.170, n^{\circ} 6$.

17 Vázquez, 1987: 195; Nieto/Sánchez, 1981: 100-101.

${ }^{18}$ Disposición para la realización de dos lámparas de plata, 13 de septiembre de 1639, Archivo Catedral, Córdoba (AC), Cuentas, 1639-2642, tomo I, caja 561.

${ }_{19}$ Nieto, 1998: 392.

${ }^{20}$ Raya/Carrillo, 2012: 500. 
no ha podido ser localizada por el momento. Mientras que la de Nuestra Señora de Villaviciosa pasa por haber sido hasta el año 1607 la capilla mayor. Una vez es consagrada la Catedral, este lugar se convertirá en un auténtico foco de devoción, debido a la colocación en su altar mayor de la Virgen de Villaviciosa y a que fue el emplazamiento escogido para instalar año tras año, el monumento al Jueves Santo ${ }^{21}$. Era normal que nobles y altos cargos eclesiásticos quisieran enterrarse allí y prodigarla de donaciones.

Las disposiciones de don Juan de Valderrama no se llevaron a cabo inmediatamente y su Obra Pía pasó a ser administrada por el propio Cabildo, a cargo de dos de sus miembros, un canónigo y un racionero, don Juan Pérez Delgado y don Luis de Castillejo. El contrato definitivo para realizarlas no se firmará hasta la década de los cuarenta, entre los dos administradores de la Obra Pía por un lado y el platero Gonzalo de Alcántara, por otro. Pocos datos se conocen aún sobre estos supervisores, salvo que don Luis era natural de Córdoba, e hijo de Andrés de Castillejo y de doña Marina de Palenzuela. Tenía un hermano que también era racionero de la Catedral, Antonio de Castillejo. Había hecho carrera universitaria y llegó a ser notario del Santo Oficio en 1630. Pertenecía a las cofradías de la Caridad, San Bartolomé, la Consolación y la Sangre 22 . La figura de don Juan Pérez Delgado permanece aún desconocida, esperando que próximos estudios aporten alguna noticia sobre él.

En 1642 los apoderados redactaran unos primeros acuerdos, en los que se fijan datos relacionados con el coste y peso de las obras: “... dichas lámparas ha de tener cuarenta y ocho marcos de plata de peso poco más o menos y por cada marco, se le ha de dar de hechura tres ducados en moneda de vellón y porque salga con toda perfección en la conformidad que estaba con los señores diputados, se le han de dar al dicho Gonzalo de Alcántara ciento y cincuenta reales demás de dicha hechura...". Se pedía que las lámparas estuvieran terminadas en julio de dicho año: “... se obligó a hacer las dichas dos lámparas de plata dentro del mes de julio próximo venidero..." ${ }^{\prime 23}$.

El 3 de enero de 1647 se vuelve a redactar otro documento entre el platero Gonzalo de Alcántara, el canónigo don Juan Pérez Delgado y el racionero de la catedral don Luis de Castillejo, en el que se puede leer: "Sepan cuantos esta carta vieren como en la ciudad de Córdoba a tres días del mes de enero de mil seiscientos y cuarenta y siete años otorgo Gonzalo de Alcántara platero de esta ciudad en la collación de Santa María que es convenido y concertado con los diputados Juan Pérez. Delgado canónigo y don Luis de Castillejo racionero de la Santa Iglesia de Córdoba como depositarios de las memorias y Obras Pías que fundó don Juan de Valderrama..., de hacer y dar hechas y acabadas dos lámparas de plata, la una para el sagrario de la dicha santa iglesia y la otra para la capilla de nuestra señora de Villaviciosa..."24. El documento de protocolos notariales reproduce de nuevo las condiciones de peso y precio de la misma, quedando estipulado que debía pesar 48 marcos de plata y que el importe de cada uno ascendía a 3 ducados por cada marco de plata. Del estudio de los legajos de protocolos y de la catedral, se ha podido establecer el total de toda la transacción, quedando desligado el coste de la suma de la plata utilizada, de la hechura de ambas y del montaje. La cuenta final se elevó a más de 11.000 reales de vellón, correspondiendo 3.445 al metal que se necesitó para su ejecución, 3.840 a la hechura y el resto al montaje: "...montará todo de plata y hechura once mil reales, diéronsele luego conforme al auto de este día con mil y cuatrocientos y treinta y seis reales y medio en plata y oro, y más cinco mil quinientos y sesenta y cuatro reales en moneda de vellón con que quedaron pagadas ambas dos lámparas..."25.

Junto con el contrato de la lámpara se halla su dibujo (Fig. 1), al que debía ceñirse para su elaboración: "firmado de las dichas partes, donde está dibujada la dicha traza y modelo porque ambas

${ }^{21}$ Raya/Carillo, 2012: 453-456.

22 Díaz, 2015: 33.

23 Acuerdo de los apoderados de Juan de Valderrama, 1642, AC, Córdoba, Cuentas, tomo I, caja 561.

${ }^{24}$ Documento de entrega de las lámparas, 3 de enero de 1647, Archivo Histórico Provincial, Córdoba (AHPC), Protocolos, oficio 25, ff. 88 y ss.

25 Coste de la bechura de la lámpara, 1642, AC, Córdoba, Cuentas, 1639-2642, tomo I, caja 561; y el resto de las disposiciones económicas están en: Documento de entrega de las lámparas, 3 de enero de 1647, AHP, Córdoba, Protocolos, oficio 25, ff. 3-4. 
lámpara han de ser iguales, del mismo peso, tamaño y hechura... "26. El diseño muestra una pieza compuesta por boya de perfil curvo, del que cuelga elemento pinjante inferior en forma de pera invertida, con toro abultado y un óvalo en resalte como único recurso decorativo. La ornamentación central consiste en otro óvalo en resalte rodeado de ces e ingredientes geométricos, a realizar con labor de repujado y cincelado. El borde superior sobresale de la zona central y se engalana con gallones abultados. Las argollas de la cadena partían de unos remates en forma de eses enroscadas. El manípulo tiene forma de plato invertido sobre el que aparece una pieza circular achatada y argolla circular. El adorno es del mismo tipo, óvalos y recursos de inspiración geométrica en realce. Estos motivos que aparecen en el manípulo se pueden ver en los nudos, en los astiles y en las esquinas de los basamentos de otras piezas que se podría relacionar a este platero o a Antonio de Alcántara, de la misma familia, y con otras muchas más de la misma época.

Como se ha mencionado, este recurso decorativo recuerda a los botones de esmalte propios de los basamentos de los ostensorios o cálices, como por ejemplo los del ostensorio de la parroquia de Nuestra Señora del Soterraño de Aguilar de la Frontera, que el profesor Moreno Cuadro relaciona con la estética de Antonio de Alcántara ${ }^{27}$. Así como también se pueden encontrar en la basa del ostensorio de la parroquia de San Francisco y San Eulogio

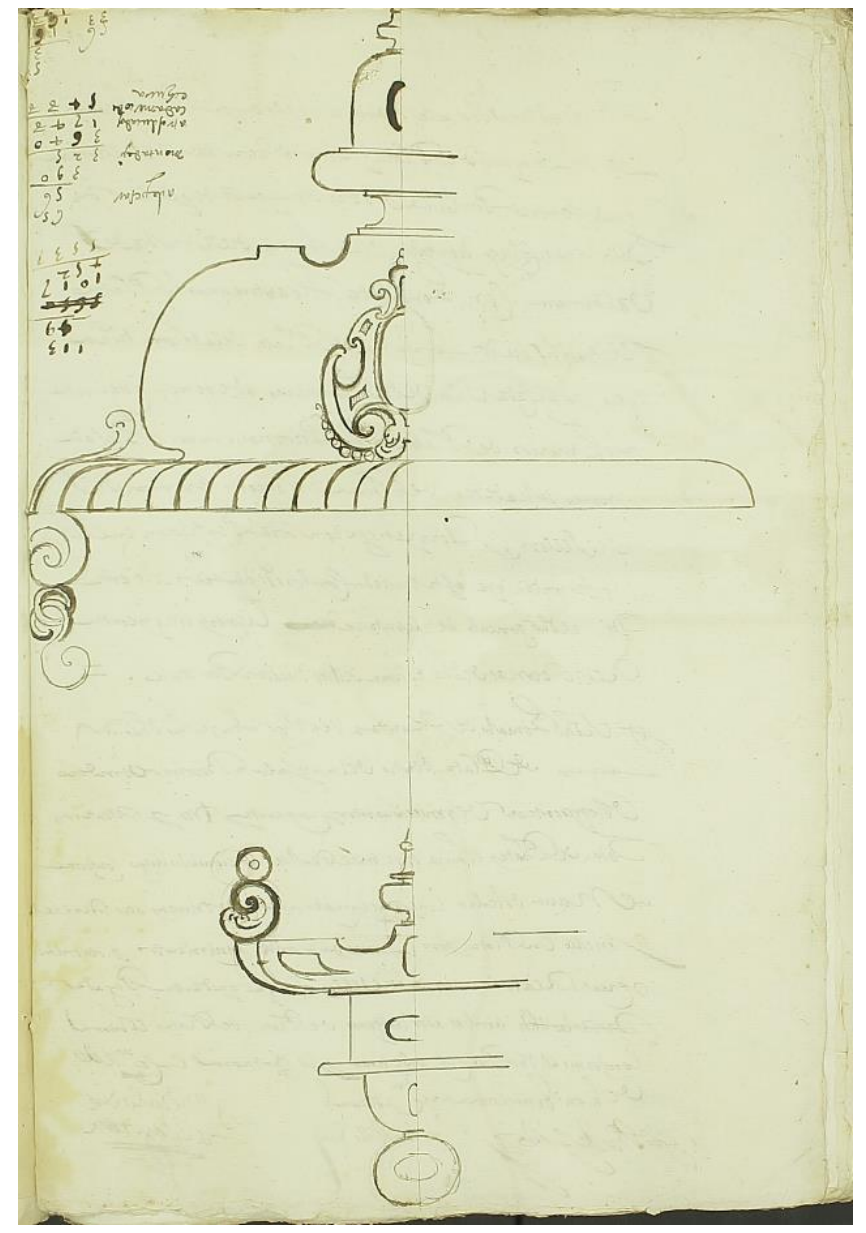

Fig. 1. Dibujo. Lámpara. Gonzalo Alcántara.

26 Papeles pertenecientes a la Obra Pía de don Juan de Valderrama y otros concernientes a ella y a la familia de los $V$ alderrama, 1647, Córdoba, ACC, Caja 563.

${ }^{27}$ Nieto/Moreno, 1993: 174. 
de la Axerquía de Córdoba ${ }^{28}$, muy posiblemente relacionado también con Antonio de Alcántara, y con los custodiados en los conventos de Santa Ana y Santa Clara de la localidad montillana ${ }^{29}$ atribuidos a dicho platero. El último de ellos es casi idéntico al del convento del Císter de Córdoba, documentado como de Antonio de Alcántara del año 1666, gracias a la inscripción que lleva en su borde ${ }^{30}$. Se reproducen de la misma manera en el pie de la custodia procesional de Cañete de las Torres, efectuada por el cuñado de Antonio de Alcántara, Simón Pérez de Tapia en 1653, del que recientemente se ha encontrado el documento contractual ${ }^{31}$. Este componente decorativo no es sólo exclusivo de los ostensorios, sino que también aparecerá en cruces procesionales, como, por ejemplo, en parte superior de la manzana del ejemplar de la parroquia de San Andrés de Córdoba, construida antes de 1644 y atribuida a Antonio de Alcántara ${ }^{32}$. Una de las lámparas que cuelgan en la capilla de Nuestro Padre Jesús Nazareno de la parroquia aguilarense lleva unos botones ovalados similares a los de este modelo, aunque el resto de la pieza es mucho más elaborado. Fue realizada para la cofradía del Santísimo Sacramento de la parroquial en el año 1660 por D. Alonso Xil Moreno, según reza en su inscripción ${ }^{33}$.

\section{Vicisitudes sobre Gonzalo de Alcántara, autor de la obra}

El apellido Alcántara fue bastante importante en el mundo de la platería cordobesa durante casi todo el siglo XVII. Se trata de una saga familiar compuesta por tres generaciones de plateros, encabezada por Gonzalo de Alcántara, su hijo Antonio, y el descendiente de este último, Tomás Gonzalo; además de un primo de Antonio, llamado también Gonzalo. Hasta el momento el más conocido es Antonio, autor de la custodia procesional de Santaella o las mazas del Ayuntamiento de Bujalance entre otras obras, y Tomás Gonzalo de Alcántara, que hizo las cruces de plata del Nazareno de Puente Genil ${ }^{34}$ y otra que se llevaron los franceses que pertenecía al Nazareno de Montilla. Con el hallazgo de los documentos aquí estudiados, se plantean una serie de nuevas incógnitas sobre esta estirpe y se abren nuevas vías de investigación.

La primera interrogante es precisamente identificar cuál de los dos Gonzalos es el autor de esta lámpara. Es difícil de resolver, ya que por el momento son escasísimos los datos encontrados sobre el primero de ellos y ninguno del segundo, salvo una noticia sobre su existencia que ofreció Valverde Madrid en un resumido artículo publicado en el Diario Córdoba en el año 1973 sobre Antonio de Alcántara ${ }^{35}$. De las referencias contrastadas documentalmente, se conoce que Gonzalo de Alcántara -padre- vivió en la collación de Santiago, donde bautiza a su hijo Antonio en $1619^{36}$ y que en fecha no conocida aún se traslada a la calle Pozo de Cueto del barrio de la Catedral, donde aparece en los padrones municipales a partir de $1640^{37}$. Tanto su hijo como su nieto residieron en la collación de Santa María. Según los legajos estudiados en este artículo, el autor de esta lámpara vivía en el año 1647 en la collación de Santa María. Ocupará cargos importantes dentro de la institución gremial. En 1623 aparece como uno de los seises, en 1632 como maestro aprobador, en 1634 y 1635 como veedor y, por último, en 1643 como alcalde ${ }^{38}$. Tuvo obrador abierto en el que aceptó el 3 de febrero de 1620 a Juan Ruiz, hijo de Benito Ruiz, como aprendiz del oficio de platero $^{39}$.

\footnotetext{
${ }^{28}$ Rodríguez, 2002: 142-141.

${ }^{29}$ Rodríguez, 2011: 220-223.

30 Dabrio, 1986: 110-111.

31 Rodríguez, 2007: 69-70.

32 Rodríguez, 2016: 93-94.

33 Ortiz, 1981: 68.

34 Rivas, 2015: 486-187.

35 Valverde, 1973b: 23.

36 Ibidem.

${ }^{37}$ Censo de Padrones, 1640, Archivo Municipal, Córdoba (AM), barrio de la Catedral, f. 3.

38 Libro primero de cabildos, 1643, AM, Córdoba, Plateros, 1591-1786, ff. 13v, 16v, 17, 17v y 19v.

39 Aceptación de un aprendiz en el obrador, 3 de febrero de 1620, AHP, Córdoba, Protocolos, oficio 4, tomo 77, f. 128 .
} 
Su marca o punzón no es conocido. Se conserva un ostensorio en el santuario de Nuestra Señora de la Sierra de Cabra, que lleva una marca ALcAN/TARA, fechado en la primera mitad del siglo XVII o el segundo cuarto de siglo, del que Ortiz Juárez dice que va acompañado del punzón de Simón de Tapia, sin poder identificar a qué Alcántara pertenece $^{40}$. Tapia no es contraste hasta 1655 , por lo que se acerca a la fecha del contrato aquí estudiado, 1647 y podría pensarse en la posibilidad de que fuera el mismo platero.

No se conocen obras documentadas de Gonzalo de Alcántara, salvo la lámpara aquí estudiada y otro contrato para la realización de unos candelabros y una cruz, cuyo destino iba a ser la Capilla de Ánimas, conocida como la capilla del Inca Garcilaso, fechado el 31 de marzo de $1631^{41}$. De su hijo Antonio también van a ser muy pocas las totalmente identificadas, y muchas las que le son atribuidas. Ya se ha mencionado anteriormente la custodia del monasterio del Císter de Córdoba fechada en 1666 y otra más, asignada que se encuentra en la parroquia de San Bartolomé de Espejo ${ }^{42}$.

Hace poco pudo ser identificada la única pieza conservada con relación documental. Se trata de una cruz procesional que se halla en la parroquia de Nuestra Señora del Soterraño de Aguilar de la Frontera. La profesora Dabrio González atribuyó a Juan Bautista de Herrera una cruz gracias a un documento contractual de la sección de protocolos del Archivo Histórico Provincial de Córdoba, realizada en 1614 y la comparaba con otra que se halla en el convento de Santa Marta de la capital, con la que hallaba similitudes, pero también notables diferencias ${ }^{43}$. Esa misma obra fue estudiada algo después, en 2016, y gracias a una carta de pago, fue relacionada con Gonzalo de Alcántara, platero de Córdoba, al que se le habrían abonado 293 reales por su ejecución ${ }^{44}$. Esto podría dar lugar a confusiones si no fuera porque en la parroquia aguilarense se encuentran dos cruces procesionales del mismo momento, con parecidos y desigualdades. El estudio profundo de las características ornamentales de Alcántara, gracias al dibujo de la lámpara de este artículo, pone en evidencia la enorme proximidad que existe entre la cruz atribuida a Gonzalo en 2016 y la pieza aquí estudiada; esta misma comparación, acerca más la otra cruz existente en dicho templo y aún sin publicar con la que Dabrio González muestra del convento de Santa Marta de Córdoba, que esta profesora dice que realizó Antonio de Alcántara.

Lejos estamos aún de poder establecer un recorrido artístico por las obras de Gonzalo de Alcántara, pero esperemos que próximas investigaciones aporten más luz al mismo y se pueda estudiar esta figura, cuáles fueron sus principales creaciones y sus características.

\footnotetext{
${ }^{40}$ Para Ortiz Juárez, esta marca es anterior a la que utilizará Antonio de Alcántara, por lo que pudiera ser la de Gonzalo. Ortiz, 1980: 81.

${ }^{41}$ Contrato para unos candelabros y una cruz, 31 de marzo de 1631, AHP, Córdoba, Protocolos, oficio 29, tomo 50, ff. 363v-366.

42 Moreno, 2006: 127.

${ }^{43}$ Dabrio, 2011: 172-174.

44 Rodríguez, 2016: 6-7.
} 


\section{Bibliografía}

Camacho Padilla, J. M.: "El Tesoro de la Catedral de Córdoba". En Boletín de la Real Academia de Córdoba de Ciencias, Bellas Letras y Nobles Artes, 31 [Núm.], Córdoba, (1931), pp. 93-102.

Cots Morató, F. de P.: El examen de maestría en el arte de plateros de V alencia: los libros de dibujos y sus artifices (1505-1882). Valencia: Delegación de Cultura. (2004).

Cruzaley Herrera, R./Ochoa Celestino, J. C.: "José Pantaleón Toscano y la lámpara mayor de la catedral de Guadalajara". En Paniagua Pérez, J. / Salazar Simarro, N. (eds.): Aurea quersoneso: estudios sobre la plata iberoamericana: siglos XVI-XIX. Universidade Católica Portuguesa, Universidad de León, Consejo Nacional para la Cultura y las Artes, e Instituto Nacional de Antropología e Historia, (2014), pp. 205-226.

Dabrio González, M ${ }^{\mathrm{a}}$ T.: "La colección de platería del Monasterio del Císter". En Apotheca, 6 [Núm.], Córdoba, (1986), pp. 107-131.

Dabrio González, Ma T: “Aportación al platero Juan Bautista de Herrera”. En Laboratorio de Arte, 23[Núm.], Sevilla, (2011), pp. 165-184.

Díaz Rodríguez, J. A.: El clero catedralicio en la España Moderna: los miembros del cabildo de la Catedral de Córdoba (1475-1808). Murcia: Universidad de Murcia. (2012).

Díaz Rodríguez, J. A.: "Diccionario biográfico de la Catedral de Córdoba (I): los miembros del cabildo en época moderna". En Historia y genealogía, 5 [Núm.], Córdoba, (2015), pp.175232.

Fresnada, F. de, obispo de Córdoba: Estatutos de la Sancta Yglesia Cathedral de Córdoba. Antequera. (1577).

García Gaínza, M. C.: Dibujos antiguos de los plateros de Pamplona. Pamplona: Universidad de Navarra. (1991).

García Zapata, I. J.: "Los dibujos de platería y su materialización: la definición de una tipología singular". En Mancini, Matteo/Pascual Chenel, Álvaro: Imbricaciones: paradigmas, modelos y materialidad de las artes en la Europa habsbuirgica. Madrid: Sílex, (2019), pp. 275-298.

López Martín, J.: La celebración eucarística, centro de la vida cristiana. Barcelona: Ed. Centre de Pastoral Litúrgica. (2005).

Martín, Fernando A.: "Dibujos de platería en la Fundación Lázaro Galdiano". En Goya: Revista de Arte, 285 [Núm.] Madrid, (2001), pp. 324-331.

Moreno Cuadro, F.: Platería cordobesa. Córdoba: Caja de ahorros y Monte de piedad de Córdoba. (2006).

Nieto Cumplido, M.: La Catedral de Córdoba. Córdoba: Publicaciones Cajasur. (1998).

Nieto Cumplido, M./Moreno Cuadro, F.: Eucharistica cordubensis. Córdoba: Monte de Piedad y Caja de Ahorros de Córdoba. (1993).

Nieto Cumplido, M./Sánchez García, L. E.: Inventario de la sección Obras Pías del Archivo de la Catedral de Córdoba: siglos XIV-XX. Madrid: Ministerio de Cultura. (1981).

Ortiz Juárez, D.: Punzones de platería cordobesa. Córdoba: Monte de piedad y caja de ahorros de Córdoba. (1980).

Ortiz Juárez, D.: "La platería cordobesa durante el siglo XVII". En Antonio del Castillo y su época. Córdoba: Diputación de Córdoba, (1986), pp. 229-251.

Ortiz Juárez, D. et allí (Coords.), Catálogo artístico y monumental de la provincia de Córdoba. I(Vol.). Córdoba: Diputación provincial de Córdoba. (1981).

Raya Raya, Ma A.: "La importancia de los inventarios en el estudio de la platería: el inventario de 1507”. En Rivas Carmona, J. (Coord.) Estudios de platería. San Eloy 2006, Murcia: Universidad de Murcia, (2006), pp. 611-629.

Raya Raya, $\mathrm{M}^{\mathrm{a}}$ A.: "La Catedral de Córdoba: un nuevo inventario del siglo XVI. Apreciaciones acerca de su realización y estudio de sus piezas más significativas". En Rivas Carmona, J. (Coord.). Estudios de platería. San Eloy 2009. Murcia: Universidad de Murcia, (2009), pp. 629-652.

Raya Raya, Ma A.: "El tesoro de la Catedral de Córdoba a través de los inventarios: un inventario de 1628”. En Rivas Carmona, J. (Coord.). Estudios de platería. San Eloy 2010. Murcia: Universidad de Murcia, (2010), pp. 628-650. 
Raya Raya, Ma A. /Carrillo Calderero, A.: "El primer inventario de El Sagrario de la Catedral de Córdoba inserto en el inventario de 1628: una praxis de dotación de un nuevo edificio". Rivas Carmona, J. (Coord.). Estudios de platería. San Eloy 2012. Murcia: Universidad de Murcia, (2012), pp. 491-508.

Raya Raya, Ma A. / Carrillo Calderero, A.: "El esplendor de la liturgia en la Catedral de Córdoba a través del inventario del 1704 (I)". En Rivas Carmona, J. (Coord.). Estudios de platería. San Eloy 2013, Murcia: Universidad de Murcia, (2013), pp. 421-443.

Raya Raya, Ma A. / Carrillo Calderero, A.: "El esplendor de la liturgia en la Catedral de Córdoba a través del inventario del 1704 (II)". En Rivas Carmona, J. (Coord.). Estudios de platería. San Eloy 2015, Murcia: Universidad de Murcia, (2015), pp. 459-476.

Raya Raya, Ma A. / Carrillo Calderero, A.: "El esplendor de la liturgia en la Catedral de Córdoba a través del inventario del 1704 (III)". En Rivas Carmona, J. (Coord.) Estudios de platería. San Eloy 2016, Murcia: Universidad de Murcia, (2016), pp. 523-541.

Raya Raya, M ${ }^{a}$ A. /Luque Carrillo, J.: "Otro inventario de platería del siglo XVIII en la catedral de Córdoba: el realizado en 1762 (I)". En Rivas Carmona, J. (Coord.). Estudios de platería. San Eloy 2018. Murcia: Universidad de Murcia, (2018), pp. 417-429.

Rivas Carmona, J.: "El impacto de la Contrarreforma en las platerías catedralicias". En Rivas Carmona, J. (Coord.). Estudios de Platería. San Eloy 2003. Murcia: Universidad de Murcia, (2003), pp. 533-534.

Rivas Carmona, J.: "La platería de la Catedral de Córdoba y su significación histórica". En Rivas Carmona, J. (Coord.). Estudios de Platería. San Eloy 2006. Murcia: Universidad de Murcia, (2006), pp. 631-650.

Rivas Carmona, J.: "Platería y exorno de la imagen: la cruz de Jesús Nazareno". En Rivas Carmona, J. (Coord.). Estudios de platería San Eloy 2015. Murcia: Universidad de Murcia, (2015), pp. 477-494.

Rodríguez Miranda, $\mathrm{M}^{\mathrm{a}}$ del A.: "La iglesia de San Francisco y San Eulogio de la Axerquía de Córdoba: algunas piezas de su orfebrería". En Peláez del Rosal, Manuel (Coord.). VI Curso de Verano San Francisco en la historia, arte y cultura española e iberoamericana. Priego de Córdoba: Ed. Asociación Hispánica de Estudios Franciscanos, (2002), pp. 139-158.

Rodríguez Miranda, $M^{a}$ del A.: "La platería en el primer señorío de los Fernández de Córdoba: Cañete de las Torres". En Ámbitos, 28[Núm.], Córdoba, (2007), pp. 65-78.

Rodríguez Miranda, $\mathrm{M}^{\mathrm{a}}$ del A.: La platería en el antiguo marquesado de Priego: Montilla (tesis doctoral). Córdoba: Repositorio Helvia. (2011), http://hdl.handle.net/10396/5557

Rodríguez Miranda, $\mathrm{M}^{\mathrm{a}}$ del A.: "La cruz procesional en Córdoba en los siglos XVI y XVII: nuevas aportaciones”. En Arte y Patrimonio, 1[Núm.], Córdoba, (2016). pp. 83-98.

Romero de Torres, E.: "La monumental lámpara de plata con incrustaciones de oro existente en la Capilla Mayor de la Santa Iglesia Catedral". En Boletín de la Real Academia de Bellas Artes de Córdoba, LV[Vol.], Córdoba, (1946), pp. 85-95.

Sánchez-Lafuente Gémar, R.: "La platería en las catedrales: del tesoro medieval a la acumulación contrarreformista". En Rivas Carmona, J. (Coord.). Estudios de platería. San Eloy 2005. Murcia: Universidad de Murcia, (2005), pp. 487-504.

Santos Márquez, A. J.: "La platería y los dibujos del pintor Juan José Del Carpio". En Rivas Carmona, J. (Coord.). Estudios de Platería San Eloy. Murcia: Universidad de Murcia, (2008). pp. 324-331.

Sanz, María J.: Antiguos dibujos de la platería sevillana. Sevilla: Diputación Provincial.

Torre y del Cerro, José de la. (1983). Registro documental de plateros cordobeses. Córdoba: Diputación provincial de Córdoba. (1986).

Valverde Madrid, J.: "El platero cordobés Pedro Sánchez de Luque". En Boletín de la Real Academia de Bellas Artes de Córdoba, 85[Vol.], Córdoba, (1963), pp. 16-74.

Valverde Madrid, J.: "Martín Sánchez de la Cruz, autor de la lámpara del Crucero de la Catedral de Córdoba". En: Diario Córdoba, 22-VII-1973, (1973a), p. 24.

Valverde Madrid, J.: “Antonio Alcántara, platero del siglo XVII”. En: Diario Córdoba, 15-VII1973, (1973b) p. 23. 
Valverde Madrid, J.: "Familiares del Santo Oficio en Córdoba (y II)". En Boletín de la Real Academia de Córdoba de Ciencias, Bellas Artes y Nobles Artes, 138[Vol.], Córdoba, (2000), p. 209.

Varas Rivero, M.: "Memoria y dibujos inéditos de una lámpara barroca de plata que perteneció al Sagrario de la Catedral de Sevilla". En Laboratorio de Arte: Revista del Departamento de Historia del Arte, 27 [Núm.], Sevilla, (2015), pp. 611-618.

Vázquez Lesmes, J. R.: Córdoba y su Cabildo Catedralicio. Córdoba: Monte de Piedad y Caja de Ahorros de Córdoba, (1987). 